\title{
sciendo
}

\author{
Current Issues in Pharmacy and Medical Sciences
}

Formerly ANNALES UNIVERSITATIS MARIAE CURIE-SKIODOWSKA, SECTIO DDD, PHARMACIA

\section{The impact of functional food on the prevention and treatment of respiratory diseases}

\author{
Gabriela Widelska ${ }^{1} \oplus$, Kamila Kasprzak-DrozD ${ }^{1}{ }^{\oplus}$,

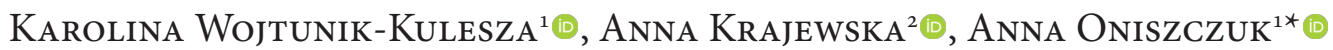

${ }^{1}$ Department of Inorganic Chemistry, Medical University of Lublin, Poland

${ }^{2}$ Department of Integrated Paediatric Dentistry, Medical University of Lublin, Poland

\section{ARTICLE INFO}

Received 18 January 2020

Accepted 10 December 2020

\section{Keywords:}

functional food,

asthma,

respiratory diseases,

antioxidants,

high altitude mountain

sickness.

\begin{abstract}
Globally, diseases of the lung are one of the main causes of death, and conventional therapies are often ineffective in dealing with this serious medical and sociological problem. Since ancient times, medicinal plants have been used in the treatment of respiratory tract diseases. Such plants show antitussive, muscle relaxing, bronchi dilation or cillary movement effects. Their usefulness has been confirmed by modern and current research. A medicinal plant that is also a functional food can open new areas in the prevention and treatment of respiratory tract diseases. In this review, information about the influence of functional food on preventing and treating asthma, chronic obstructive pulmonary disorder (COPD) and high-altitude sickness are presented.
\end{abstract}

\section{INTRODUCTION}

In recent times, functional food is becoming increasingly popular globally and not without reason. The first information about nutraceuticals comes from 1980, when Japanese researchers, in following the science and philosophical tradition of the Far East, abandoned a clear separation between food and medicine. At that time, the definition of functional food was also established. Accordingly, food has a natural origin, and due to specific modifications, they can be used for preventing and treating diseases, protecting the health, and extending the patient's lifepan. Food, in order to be considered as functional, must necessarily have health-promoting effects (apart from the obvious nutrition functions) confirmed by numerous and reliable controlled studies [1]. Additionally, these foods should also be similar to conventionals, and, what is very important, the product should have its beneficial effects at the recommended dosage corresponding with standard consumption. What is worth mentioning is that functional food (not like dietary supplements - capsule, tablet, or powder) is derived from natural components and should be consumed as part of the proper, daily diet. The beneficial properties of functional food can be used against cancers, coronary heart disease, high blood pressure, respiratory disease, inflammations, viral infections and civilizational diseases (different kinds od dementions, cardiovascular diseases e.g.). Functional food supports the

\footnotetext{
* Corresponding author

e-mail: annaoniszczuk@umlub.pl
}

treatment of existing diseases, promotes physical and mental well-being, and inhibits the aging process [2]. Many plant extracts can activate mucous that forms along the respiratory tract to combat microbes and make the removal of unwanted foreign bodies much easier. In functional food, the plant constituents that provide both taste and food aroma can be also used as expectorant antitussive antimicrobial and irritation soothers in the respiratory system. Thanks to appropriate modifications, functional food may increase mucin secretion, eliminate numerous pathogens, reduce inflammation, and also by tearing apart hydrogen bonds, can decrease the viscosity of the mucus secreted into the respiratory tract [2].

\section{THE IMPACT OF FUNCTIONAL FOOD ON THE DEVELOPMENT AND TREATMENT OF ASTHMA}

Asthma is a heterogeneous disease, most often characterized by chronic inflammation of the respiratory tract that is associated with wheezing, shortness of breath, and coughing. The existing classification indicates extrinsic (socalled atopic) and intrinsic (non-atopic) forms of asthma. In the first case, the inflammatory process is initiated by the reaction of allergens with IgE antibodies, located on mast cells and basophils receptors. Bronchoconstrictor mediators and proinflammatory cytokines are secreted, this in turn stimulates the migration of eosinophils and lymphocytes to the local area of inflammation. However, the impact of allergens and $\mathrm{IgE}$ on the formation of non-atopic asthma 
has not been demonstrated [3]. Some irregularities in the cytokine profile were also observed. Furthermore, a reduction in the release of interleukin 12 (IL-12) by macrophages was noted, and subsequently, the advantage of differentiation of Th0 lymphocytes into Th2 lymphocytes, triggering excessive amounts of interleukin 4 and 5 (IL-4 and IL-5) and simultaneously caused deficiency of Th1 lymphocytes. Some scientists speculate that the main reason for changes in the phenotype of Th0 lymphocytes in Th2 is increasingly rare exposure to any respiratory infections in childhood [4]. It has also been shown that atopic asthma may resemble nonatopic asthma over time. The whole inflammatory process does not depend so much on contact with the allergen, but on the mediators released from pro-inflammatory cells.

Asthma is currently divided into 4 grades of GINA severity [4]:

Episodic asthma - with short attacks less than 1 time a week, night attacks less than 1 time a month, no symptoms between exacerbations. Peak expiratory measurement (PEF) is above $80 \%$ of predicted norm, PEF fluctuations are below $20 \%$, with normal PEF after diastolic drug administration. Seizures are closely related to occasional exposure to a specific allergen particle, recognized by $\mathrm{E}$ antibodies or $\mathrm{T}$ cells in the air, food and environment. Skin tests may help patients to „decode" the cause of the seizure, if they know about the nature of their own unique allergy and note the circumstances of 2 subsequent incidents.

Mild chronic asthma - with short attacks more than 2 times a week, night attacks more than 2 times a month, no symptoms between exacerbations. PEF is above $80 \%$ predicted, fluctuations 20-30\%, and shows normal PEF after diastolic drug administration. The pattern depends on frequency of contact with the allergen. Skin tests can still make it easier for a patient to ,find" the cause, if they are convinced of the nature of their allergy and can make a note of several subsequent events. The occurrence of seizures after supper is characteristic, hence the nocturnal symptoms. Performing tests can help to „discover" the cause of a seizure if the afflicted can identify which component of their daily diet is pathogenic. Since tests with standardized food allergens are often false-negative, fresh produce testing should be performed.

Severe chronic asthma - with frequent day and night attacks, constant symptoms, limited physical activity, hospitalization in the last year and life-threatening attacks. Here, $\mathrm{PEF}$ at resting is below $60 \%$ with fluctuations above $30 \%$ and being abnormal despite medication. The pattern depends on the constancy of contact with the alergen [4,5].

An asthma attack is often accompanied by wheezing, tightness of the chest, shortness of breath and coughing, all of which may regress spontaneously or after use of appropriate medicines. Most commonly, in asthma therapy, corticosteroids as an oral inhalation is used. Clinical trials conducted on the possibility of using herbal medicines have shown a significant improvement on sleep quality, reduction in the amounts of coughing attacks and their intensity in patients with recognized asthma of allergic etiology who regularly use herbal infusions (containing chamomile, anise, cumin, saffron, fennel, black cumin) compared to those receiving placebo [5]. Diet and nutrition can significantly have an impact on creation and development of chronic inflammation in the respiratory system. As stated in the study, asthmatics have notably lower lycopene and vitamin A levels in blood serum, compared to control groups of healthy individuals [6]. Moreover, it has also been shown that a diet rich in fish or functional food enriched with omega-3 fatty acids has a protective effect on the respiratory system in the case of allergic asthma. In particular, antiinflammatory effects of oleic and hexadecanoic acid were observed, which indicates that these can be successfully used in the treatment of asthma [7]. In addition, it has been observed that eating fresh fruit rich in vitamin $\mathrm{C}$ reduces the incidence of asthma and improves respiratory function in children (Swee-Ling and Suahila 2016).

An extract obtained from Euphorbia hirta L. may be a promising food additive. It contains phytol along with its isomer (3,7,11,15-tetramethyl-2-hexadecen-1-ol), essential oils, alkaloids, tannins and saponins. This plant is being used successfully to treat asthma and lung diseases in Africa. Moreover, the plant contains vitamin $\mathrm{K}$ and $\mathrm{E}$ - popular antioxidants that prevent the development of inflammation in the respiratory tract [8]. A study has shown that phytols found in the extracts are produced by the hydrolysis of chlorophyll.

Another plant, Nigella sativa L. (black cumin) also demonstrates anti-asthmatic effects. In randomized control studies, 29 patients with asthma were tested. Fourteen individuals served as a control group, while fifteen people were treated with $0.1 \%$ Nigella sativa extract at a dose of $15 \mathrm{mg} / \mathrm{kg}$ body weight. Significant improvement (decreasing number of asthmatic attacks, no wheezing) were seen in the patients using the extract compared to the control group [8].

Asystasia gangetica L. is a very popular medicinal plant in Nigeria and was tested for its spasmolytic and anti-inflammatory properties. Its potency was found to be dependant on the solvent used for extraction. Other plants with proven anti-asthmatic potential are Allium sativum (garlic), Allium ascolanicum (shallot), Anacardium occidentalis (cashew), Zingiber officinale (ginger), Mimosa pigra (mimosa), Eugenia aromatica (clove). All of these can be a rich source of extracts and components for functional foods use [9].

\section{ENRICHED FOODS AND CHRONIC OBSTRUCTIVE PULMONARY DISEASE (COPD)}

Chronic obstructive pulmonary disease (COPD) is a major respiratory disease and the third leading cause of death in the world after coronary heart disease and stroke. The symptoms of this clinical condition manifest by airway obstruction and chronic respiratory failure combined with permanent inflammation. Chronic obstructive pulmonary disease can be often caused by toxins inhalation, including those derived from cigarette smoke. These toxins induce chronic inflammation of the respiratory system and alveoli, followed by chronic bronchitis and bronchiolitis, and ultimately emphysema. Moreover, hyperplasia of the glands secreting mucus into the respiratory tract, inflammatory infiltration and pulmonary fibrosis can occur.

Fruits and vegetables rich in vitamins $\mathrm{C}$ and $\mathrm{E}$ can be useful in preventing and treating COPD. Research has noticed that smokers with high oxidative stress are more 
susceptible to antioxidant supplementation. For non-smokers, the daily protective dose of vitamin $\mathrm{C}$ is $60 \mathrm{mg} /$ day, while for smokers it is $100 \mathrm{mg} /$ day [10]. The contained kaempferol, isorhamnetin, quercetin, engelerin and caffeine n-butyl ester of the the genus Similax, as well as the gingerol obtained from ginger Zingiber officinale were found to inhibit activation of factor NF-kB, which is involved in the formation of the inflammatory process in the respiratory system [1]. In addition, the curcumin present in Curcuma longa was shown to be effective in the experimental treatment of bronchitis and emphysema. Its effect on the reduction of COPDspecific molecular parameters, the amount of oxidants, and the extent of inflammation have been underlined [3].

Traditional Chinese Medicine is a valuable source of plant material for the production of functional food. The effectiveness of many plant extracts (schizander, crotch, ginger, ginseng) has been reported. These are known to enhance the antigen-specific antibodies located in the respiratory mucus and to increase the resistance of the respiratory tract itself, as well as to reduce inflammation [1]. The traditional medicine of India and Mexico can also be extensively mined for functional food uses. For instance, Ocimum sanctum L. (holy basil) is useful in the treatment of bronchitis and cold; Argemone ochroleuca L., because of its berberine content, relaxes tracheal muscles and this is helpful in the treatment of COPD; Thyme extract (Thymus vulgaris L.) inhibits endothelin-induced tracheal contraction which also benefits asthma and COPD patients [11].

\section{IMPACT OF DIET ON THE PREVENTION OF ALTITUDE SICKNESS}

A common definition of mountain sickness was established during the Lake Louise Conference international symposium, where the main theme concerned various aspects of hypoxia and its impact on the human body. At that time, all known symptoms of the high-altitude disease were collected and adjusted to severity (Table 1) [12].

Table 1. Lake Louise criteria [12]

\begin{tabular}{|c|c|}
\hline Condition & Symptoms \\
\hline $\begin{array}{l}\text { Acute Mountain Sickness } \\
\text { (AMS) }\end{array}$ & $\begin{array}{c}\text { Headache, breathlessness, insomnia, } \\
\text { nausea, anorexia }\end{array}$ \\
\hline $\begin{array}{l}\text { High-Altitude Cerebral Edema } \\
\text { (HACE) }\end{array}$ & $\begin{array}{c}\text { Changes in mental state or motor } \\
\text { coordination, people with already } \\
\text { developed or not developed acute high } \\
\text { mountain disease }\end{array}$ \\
\hline $\begin{array}{l}\text { High-Altitude Pulmonary Edema } \\
\text { (HAPE) }\end{array}$ & $\begin{array}{l}\text { At least two of the following symptoms: } \\
\text { sleep apnoea, weakness or decrease } \\
\text { physical endurance, chest tightness or } \\
\text { embolism, and auscultatory crackling or } \\
\text { wheezing in at least one lung, cyanosis, } \\
\text { rapid breathing, increased heart rate }\end{array}$ \\
\hline
\end{tabular}

Altitude sickness is mainly caused by reduced oxygen availability as a result of lowered atmospheric pressure (hypobaric hypoxia). Despite the fact that the percentage of oxygen in the atmosphere is constant (about 21\%), as a result of the altitude increase, the atmospheric pressure decreases, and the air becomes increasingly thinner. All this in a short time and without proper physical preparation of the body gives symptoms of hypoxia. The most effective method of adapting to the low-pressure conditions is an appropriate acclimatization, sometimes lasting even several weeks. Persons whose acclimatization did not proceed properly or was intentionally abandoned are exposed to various types of altitude diseases, including acute mountain sickness (AMS Acute Mountain Sickness), high altitude pulmonary edema (HAPE - High-Altitude Pulmonary Edema), high altitude brain edema (HACE - High-Altitude Cerebral Edema) or the much milder 'alpine headache'. Because each organism is different, climbers also react differently to changes in altitude. Hence, it is very difficult to predict who will get symptoms and who will not. The course consists of acclimatization - whole series adaptation processes that must carry the body to become accustomed to the reduced oxygen content in the blood.

Through knowledge of genomics, proteomics, metabolomics, transcriptomics, it becomes easier to understand the mechanism by which high-altitude diseases are caused.

It has been shown that hyperbaric hypoxia, associated with mountain anorexia and impaired intestinal absorption, induces weight loss. The level of oxygen saturation in the body is also significant, saturation about $70 \%$ is necessary for maintaining adequate absorption in the small intestine. In the past, scientists have repeatedly argued over the role of carbohydrates during acclimatization - whether it have an impact on fat metabolic activity, if the carbohydrate level depends on the altitude, and whether extensive physical exercise at sea level has a positive effect on alpine sickness. There is little evidence that prolonged (or sudden) exposure to high activity increases the demand for a particular micronutrient, although there are reports of the benefits of additional iron and vitamin E supplementation [13]. Studies on the effects of temperature and energy expenditure, however, suggest that in the case of exposure to ultraviolet radiation with a reduced oxygen level in the atmosphere, supplementation with antioxidants would be indicated. So, what would happen if all these ingredients were supplied via food intake rather than by the traditional vitamin tablets?

At high altitudes, prolonged consumption of vitamins and other substances with antioxidant properties can prevent the slowing of blood flow and tissue changes due to free radical release [14]. Effective usage of functional foods can aid in maintaining or increasing blood flow, as well as positively affecting the deformability of the red blood cell membrane, thus improving oxygen transport to tissues, hence having beneficial effects on the body acclimatizing to mountain altitudes. The dietary mechanisms affecting membrane fluidity include supplementation with polyunsaturated fatty acids and carbohydrate. Moreover, it also seems reasonable to supplement the diet with iron preparations that support the synthesis of hemoglobin at high altitudes [15].

In 2002, a group of Spanish climbers attempted to climb the $710 \mathrm{~m}$ high Jannu Mountain in the Himalayas. The aim of the study was to analyze and evaluate the diet used by the climbers. All participants of the expedition aged 27 to 42 years had considerable mountain experience and were in excellent physical condition. The experiment showed that the climber's daily caloric demand was in the range of 3800-6000 kcal depending on the physical activity carried out during the climbing.

Observed weight loss (15\% at an altitude of 5300-8000 m) can significantly affect the weakening of both mental and physical conditions [13]. Some studies suggest that taking 
in a certain amount of carbohydrates improves blood oxygenation and provides a more efficient source of energy compared to fats and protein. Consuming proteins at the appropriate, recommended level, in combination with liquid glucose, was shown in research to have a positive effect on the proper functioning of the body in high catabolism situations. As demonstrated, the diet used during the Jannu mountain trip (rich in fats, low in carbohydrates) was not conducive to acclimatization, because the metabolism of excessive amounts of fats required increased oxygen supply, which in turn was at the expense of hypoxia of other tissues. It should be noted that insufficient carbohydrate intake results in weaker muscle strength and liver regeneration, leading to hyperglycemia, protein catabolism and weight loss.

Some scientists have conducted research into the use of antioxidants as anti-inflammatory and oxidative stressreducing substances. Among the plants that were researched, special attention was given to Ginkgo biloba (Ginkgo biloba). The use of ginkgo biloba as an ingredient of functional food for athletes was found to be most justified. Its strong influence on blood vessel conditions (including cerebral vessels) and impact on cognitive functions and memory has been known for centuries. In addition, it was found to inhibit the formation of blood clots that could result in serious damage to health or death for people staying at high altitudes in states of chronic hypoxia [16].

Subsequent reports about the effectiveness of natural substances in the process of acclimatization made reference to studies on the use of the popular Mediterranean plant Sideritis scardica or Iron plant. Traditionally, infusions from the flowers and stems of this interesting plant have been considered a panacea for many diseases. Investigations demonstrate that it has anti-inflammatory, antiseptic and soothing effects. More importantly, the extract was found to be helpful in all diseases related to respiratory failure. In addition, infusions of iron plant was discovered to lower blood pressure, which has a positive effect on the entire circulatory system and is desirable during acclimatization. It also has antioxidant effects and prevents the development of oxidative stress at significant heights.

The chemical composition of sideritis extract also deserves attention as it contains, among others, flavonoids, diterpenes, steroids, iridoids, coumarins, lignans and essential oils [17]. The presence of polyphenols, including hydroxy-cinnamic acid, is most likely responsible for the beneficial effects in acclimatization, namely, enabling an increase in blood flow to the brain, an increase in oxygen saturation, a decrease in height-induced headache, and an increase in climber's cognitive functions. The action mechanism of the extract is related to the ability of polyphenols to prevent the reuptake of monoamine neurotransmitters. Researchers also compared effects of Sideritis scardica extract with Gingko biloba extract. Compared to the control group receiving placebo, the group treated with sideritis extracts showed better and faster recovery time and the ability to exert greater effort. What is more, Sideritis scardica was found to be more effective when tested for hemoglobin oxygenation enhancement and increased oxygen saturation in the prefrontal cortex during cognitive tests [18]. Therefore, thanks to its properties, this plant could be a perfect material for the production of functional food supporting acclimatization.

Bee bread is another noteworthy substance. It is a substance produced by bees and it is a complex of pollen mixed with honey and preserved by bees in a fermentation process. The chemical composition of bee bread is extremely rich and contains proteins (10-40\%), carbohydrates (fructose and glucose), saturated and unsaturated fats (phospholipids and phytosterols), polyphenolic compounds (flavonoids, phenolic acids, leukotrienes, catechins), triterpenic compounds (oleanate and ursulic acid), as well as huge deposits of micro- and macroelements. Its strengthening effect has been recognized by climbers for many years. In addition, it has been shown to stimulate the haematopoietic system to produce hemoglobin and erythrocytes, even in cases where iron supplementation were not effective. This property is extremely valuable for acquired acclimatization in the high mountains [19].

\section{CONCLUSIONS}

Globally, respiratory diseases are one of major causes of death. Unfortunately, modern medicine cannot completely heal many of the afflicted. Ancient literature and traditional medicine can help to solve this problem. Since time immemorial, plants have been successfully used in treating respiratory diseases due to their antitussive, expectorant, spasmolytic and antipyretic properties. Thanks to our knowledge of science, we can focus on acquiring specific compounds for specific purposes. To avoid the use of classic medicines, the an emphasis can be placed on prevention. Functional foods, in addition to preventive action, can support the treatment of already existing diseases.

\section{CONFLICT OF INTEREST}

The authors have no conflict of interest to declare.

\section{ORCID iDs}

Widelska Gabriela Dhttps://orcid.org/0000-0002-7663-2827 Kamila Kasprzak-Drozd

(D)https://orcid.org/0000-0002-6282-6313

Karolina Wojtunik-Kulesza

(1) https://orcid.org/0000-0002-6541-8817

Anna Krajewska (Dhttps://orcid.org/0000-0001-9699-1344

Anna Oniszczuk (Dhttps://orcid.org/0000-0002-5109-3302

\section{REFERENCE}

1. Swee-Ling L, Suahila M. Functional food and dietary supplements for lung health. Trends Food Sci Technol. 2016;57:74-82.

2. Pearson W, Charch A, Brewer D, Clarke AF. Pilot study investigating the ability of an herbal composite to alleviate clinical signs of respiratory disfunction in horses with recurrent airway obstruction. Can J Vet Res. 2007;71(2):145-51.

3. Ram A, Balachandar S, Vijayananth P, Singh VP. Medicinal plants useful for treating chronic obstructive pulmonary disease (COPD): Current status and future perspectives. Fitoterapia. 2011;82(2):141-51.

4. Boskabady M, Javan H, Sajady M, Rakhshandeh H. The possible prophylactic effect of Nigella sativa seed extract in asthmatic patients. Fundam Clin Pharmacol. 2007;21(5):559-66. 
5. Haggag EG, Abou-Moustafa MA, Boucher W, Theoharides TC. The effect of a herbal water-extract on histaminę release from mast cells and on allergic asthma. J Herb Pharmacother. 2003;3(4):41-54.

6. Riccioni G, Bucciarelli T, Mancini B, Di Ilio C, Della Vecchia R, D'Orazio N. Plasma lykopene and antioxidant vitamins in asthma: the PLAVA study. J Asthma. 2007;44(6):429-32.

7. Ruxton CHS, Cakler PC, Reed SC. The impact of the long-chain n-3 polyunsaturated fatty acids on human health. Nutr Res Rev. 2005;18(1):113-29.

8. Hultqvist M, Olofsson P, Gelderman K. A new arthritis therapy with oxydative burst inducers. PLoS Med. 2006;3(9):1-17.

9. Akah P, Ezike A, Nwafor S Evaluation of the anti-asthmatic property of Asystasia gangetica leaf extracts. J. Ethnopharmacol. 2003;89(1):25-36.

10. Romieu I, Trenga C. Diet and obstructive lung diseases. Epidemiol Rev. 2001;23(2):268-87.

11. Sanchez-Mendoza M, Castillo-Henkel C, Navarrete A. Relaxant action mechanism of berberine identified as the active principle of Argemone ochroleuca Seet in Guinea-pig tracheal smooth muscle. J Pharm Pharmacol. 2008;60(2):229-36.

12. Roeggla G, Roeggla M, Podolsky A, Wagner A, Laggner AN. How can acute mountain sickness be quantified at moderate altitude? J R Soc Med. 1996;89(3):141-3.

13. Mariscal-Arcas M, Carvajal C, Monteagudo C, Lahtinen J, Fernandez de Alba MC, Feriche B, Ollea-Serrano F. Nutritional analysis of diet at base camp of a seven thousand-metre mountain in the Himalayas. Rev Andal Med Deport. 2010;3(4):127-32.
14. Bailey DM, Davies B, Milledge JS, Richards M, Williams SR, Jordinson M, Calam J. Elevated plasma cholecystokinin at high altitude: metabolic implications for the anorexia of acute mountain sickness. High Alt Med Biol. 2000;1:9-23.

15. Berglung B. High-altitude training. Aspects of haematological adaptation. Sports ed. 1992;14:289-303.

16. Jafarian S, Gorouhi F, Salimi S, Lotfi J. Sumatriptan for prevention of acute mountain sickness: randomized clinical trial. Ann Neurol. 2007;62(3):273-7.

17. Tadic VM, Jeremic I, Dobric S, Isakovic A, Markovic I, Trajkovic V, Bojovic D, Arsic I. Anti-inflammatory, gastroprotective and cytotoxic effects of Sideritis scardica extracts. Planta Med. 2012;78(5):415-27.

18. Wightman E, Jackson P, Khan J, Forster J, Heiner F, Feistel B, et al. The acute and chronić cognitive and cerebral blood flow effects of a Sideritis scardica (greek mountain tea) extract: a double blind, randomized, placebo controlled, parallel groups study in healthy humans. Nutrients. 2018;10:955-78.

19. Ozcan MM, Aljuhaimi F, Babiker EE, Uslu N, Ceylan DA, Ghafoor K, et al. Determination of antioxidant activity, phenolic compound, mineral contents and fatty acids compositions of bee pollen grains collected from different locations. J Apic Sci. 2019;63(1):69-79. 